\title{
Multiple combined indirect procedure for the surgical treatment of children with moyamoya disease. A comparison with single indirect anastomosis with direct anastomosis
}

\author{
Toshio Matsushima, M.D., Takuya Inoue, M.D., Kiyonobu Ikezaki, M.D., Koichiro Matsukado, \\ M.D., Yoshihiro Natori, M.D., Takanori Inamura, M.D., and Masashi Fukui, M.D. \\ Department of Neurosurgery, Neurological Institute, Faculty of Medicine, Kyushu University, Fukuoka, \\ Japan
}

Considering three different bypass procedures now in use, (single indirect nonanastomotic bypass procedure, multiple combined indirect (MCI) nonanastomotic procedure and direct anastomosis), the authors attempted to identify the most appropriate bypass procedure for treating ischemic-type moyamoya disease in children.

The authors performed three procedures (the original encephaloduroarteriosynangiosis [EDAS] alone, the frontotemporoparietal combined indirect bypass procedure, and the superficial temporal artery-middle cerebral artery [STA-MCA] anastomosis with encephalomyosynangiosis [EMS]) on 72 hemispheres in 50 patients with pediatric moyamoya disease. Analyses were then performed to compare postoperative collateral vessel formation found on angiograms, complications, and clinical improvements. Postoperative collateral formations were observed in more than two-thirds of the MCA distribution after the EDAS alone, the MCI procedure, and the direct anastomosis in $44 \%, 52 \%$, and $74 \%$ of the surgically treated hemispheres, respectively. In addition, frontal encephalomyoarteriosynangiosis of the MCI bypass procedure formed collateral vessels of the anterior cerebral artery distribution in $94 \%$ of the treated hemispheres. Postoperatively, clinical symptoms resolved in $56 \%, 63 \%$, and $74 \%$ of the treated sides 1 year after EDAS alone, MCI procedure, and the direct anastomosis, respectively. One patient suffered a minor stroke after EDAS alone, two patients developed epidural hematomas after the MCI procedure, and one patient suffered a major stroke and one patient a minor stroke after undergoing direct anastomosis.

The direct anastomosis procedure was found to result in the best postoperative collateral vessel formation and clinical improvement. However, the single and multiple combined indirect nonanastomotic bypass procedures were found to be safer than direct anastomosis. Furthermore, the frontotemporoparietal combined indirect bypass procedure caused the formation of collateral circulation not only in the MCA but also in the ACA distribution. Based on analysis of these findings, the authors recommend the MCI procedure as the appropriate surgical procedure in the treatment of children with moyamoya disease, 
although the best treatment is the STA-MCA anastomosis with EMS when feasible.

Key Words * moyamoya disease * surgical treatment * indirect nonanastomotic bypass procedure * multiple combined indirect procedure * frontotemporoparietal combined indirect bypass procedure * child

Most pediatric patients with ischemic-type moyamoya disease first present after suffering a transient ischemic attack (TIA) and later develop cerebral infarction.[9] The goal of surgical treatment in such patients is to establish adequate and sufficient collateral circulation to prevent cerebral infarction.

Various bypass procedures, including direct and indirect anastomoses, have been performed; however, controversy remains with regard to the most suitable procedure.[6,16,18,19,24,27,29] Each procedure has its own drawbacks.[12,13,16,20,24,27] To overcome these problems we developed a combined frontotemporoparietal indirect bypass technique as a multiple-combined indirect (MCI) procedure.[11,15-19] In this report we compare the surgical results obtained using the MCI procedure with results obtained using original encephaloduroarteriosynangiosis (EDAS) alone and results obtained using superior temporal artery-middle cerebral artery (STA-MCA) anastomosis with encephalomyosynangiosis (EMS) to clarify the advantages and disadvantages of each procedure.

\section{CLINICAL MATERIAL AND METHODS}

At Kyushu University Hospital, 50 children with moyamoya disease who presented mainly with ischemic symptoms underwent surgery in 72 hemispheres. Clinical features included TIA, cerebral infarction, and epilepsy, and there were no cases of hemorrhage (Table 1).

\begin{tabular}{|ccccccccc|}
\hline \multicolumn{10}{c|}{ TABLE 1 } \\
ClINICAL FEATURES OF 50 PATIENTS WHO UNDERWENT ONE OF THREE PROCE- \\
DURES FOR THE TREATMENT OF MOVAMOVA. DISEASE
\end{tabular}

The patients ages ranged from 1 to 16 years. All surgeries were performed using intraoperative $\mathrm{PaCO}_{2}$ monitoring. Each patient underwent one of three different procedures: the original EDAS alone as a single indirect procedure from 1983 to 1986; the STA-MCA anastomosis with EMS as direct anastomosis (1987-1993); and the MCI bypass procedure (1990-1996) (Figs. 1 and 2). 


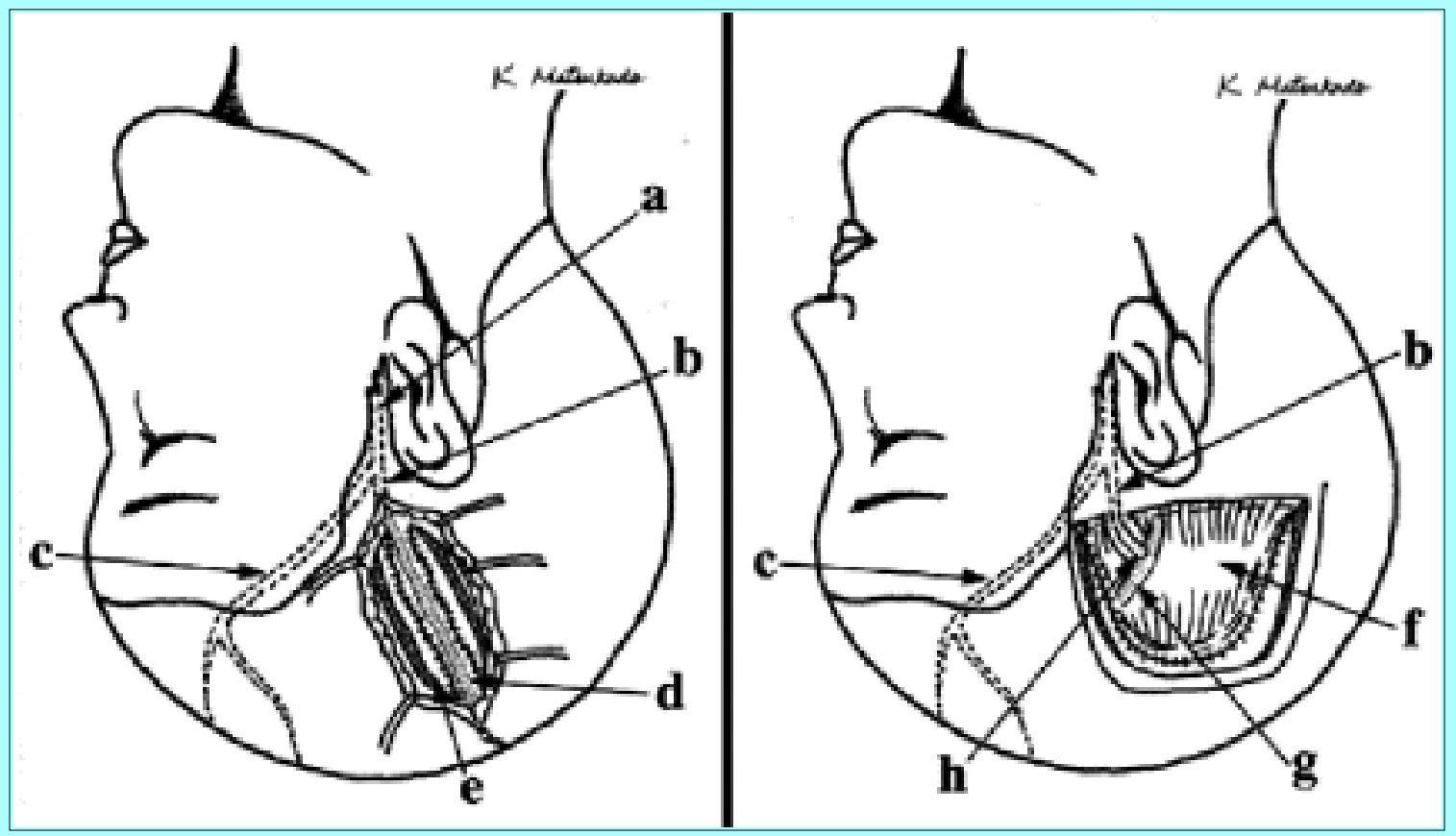

Fig. 1. Illustrations depicting the operative procedures of the original EDAS and STA-MCA anastomosis with EMS. Left: Original EDAS. The posterior branch of the STA is exposed with the surrounding galea by using the cut-down technique. After craniotomy, the edges of the galeal strip with the branch are sutured to the edges of the linear dural incision. The bone flap is reflected back and over. Finally, the STA is revealed to course on the brain surface under the skull. Right: The STA-MCA anastomosis with EMS. The STA-MCA anastomosis is performed, and then the operative field over the anastomosis is covered by the muscle flap instead of the dura mater. a: main trunk of the STA, b: posterior branch of the STA; c: anterior branch of the STA; d: galeal flap; e: dura mater, f: temporal muscle; g: branch of the MCA; h: anastomosis. 


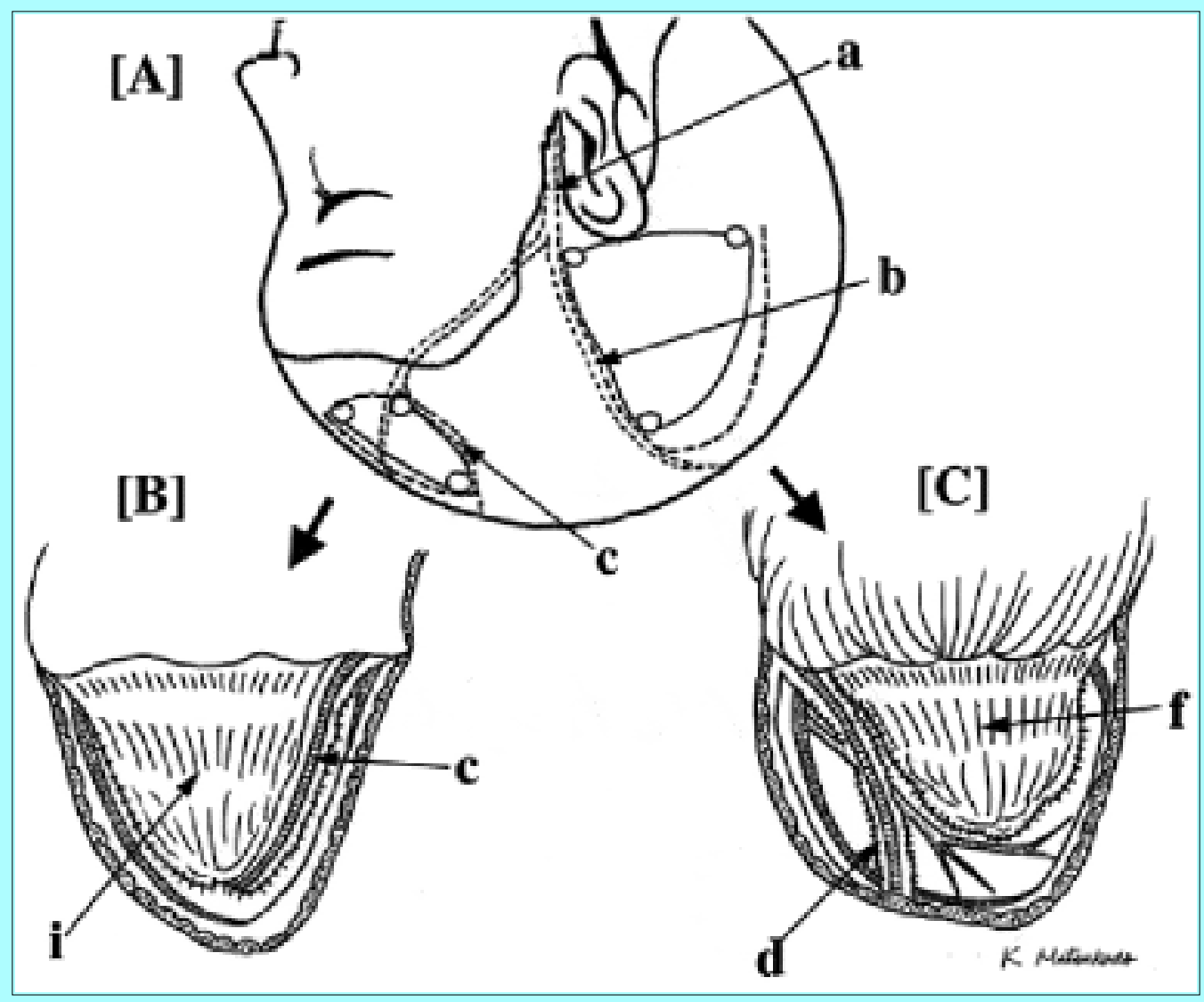

Fig. 2. Illustrations showing the operative steps in the frontotemporoparietal combined procedure. A: Depiction of the relationship between the courses of the anterior and posterior branches of the STA and the skin incisions and craniotomies. The courses of the anterior and posterior STA branches are shown, and the skin incisions are related to the courses of the branches. The locations of the frontal and temporoparietal craniotomies are also demonstrated. B: Depiction of the frontal encephalomyoarteriosynangiosis. After frontal craniotomy, the dura mater is partially removed, and the dural defect is covered with the frontal muscle with the anterior branch of the STA. C: The EDAS and EMS in the temporoparietal bony opening. After a temporoparietal craniotomy, EDAS by using the posterior branch of the STA is performed with EMS in the same large bony opening. $\mathrm{i}$ : frontal muscle and periosteum; see Fig. 1 for definitions.

When we began to treat pediatric patients surgically in 1983, we usually chose to perform the original EDAS alone because it is a less invasive procedure. However, we later changed this policy because occasionally either no or only poor collateral circulation was obtained, and reoperation was therefore required in some cases.[12] Thereafter we performed the direct bypass procedure, and we have compared the results with those after performing the EDAS alone.[18] On the other hand, we also attempted to identify the most effective combination of various indirect procedures, and eventually came to perform the frontotemporoparietal combined indirect procedure in pediatric patients. $[11,15,17,19]$ In most patients the bypass surgery was performed bilaterally, whereas in those patients with a unilateral symptom the procedure was confined to one side only. Eighteen hemispheres in 12 patients were surgically treated using the original EDAS alone, 35 hemispheres in 22 patients by using the MCI bypass procedure, and 19 hemispheres in 16 patients by using direct anastomosis (Table 1). Patients were followed for longer than 1 year.

In all patients, selective angiography was performed at 4 to 25 months postsurgery. We evaluated and 
compared the postoperative collateral formation results for the three different procedures, using the lateral view on serial external carotid artery angiograms. The extent of collateral formation after each procedure was evaluated as either the whole, over two-thirds, over one-third, or none of the MCA distribution (Fig. 3). In the patients who underwent the MCI procedure, the extent of collateral circulation formed was evaluated in the MCA distribution through the temporoparietal EDAS and EMS, and in the anterior cerebral artery (ACA) distribution through the frontal EMAS. Clinical follow-up results 1 year postsurgery were classified into three groups in which the symptoms had either completely disappeared, decreased to some extent, or were unchanged. These results, including postoperative complications, were finally compared among the three procedures.

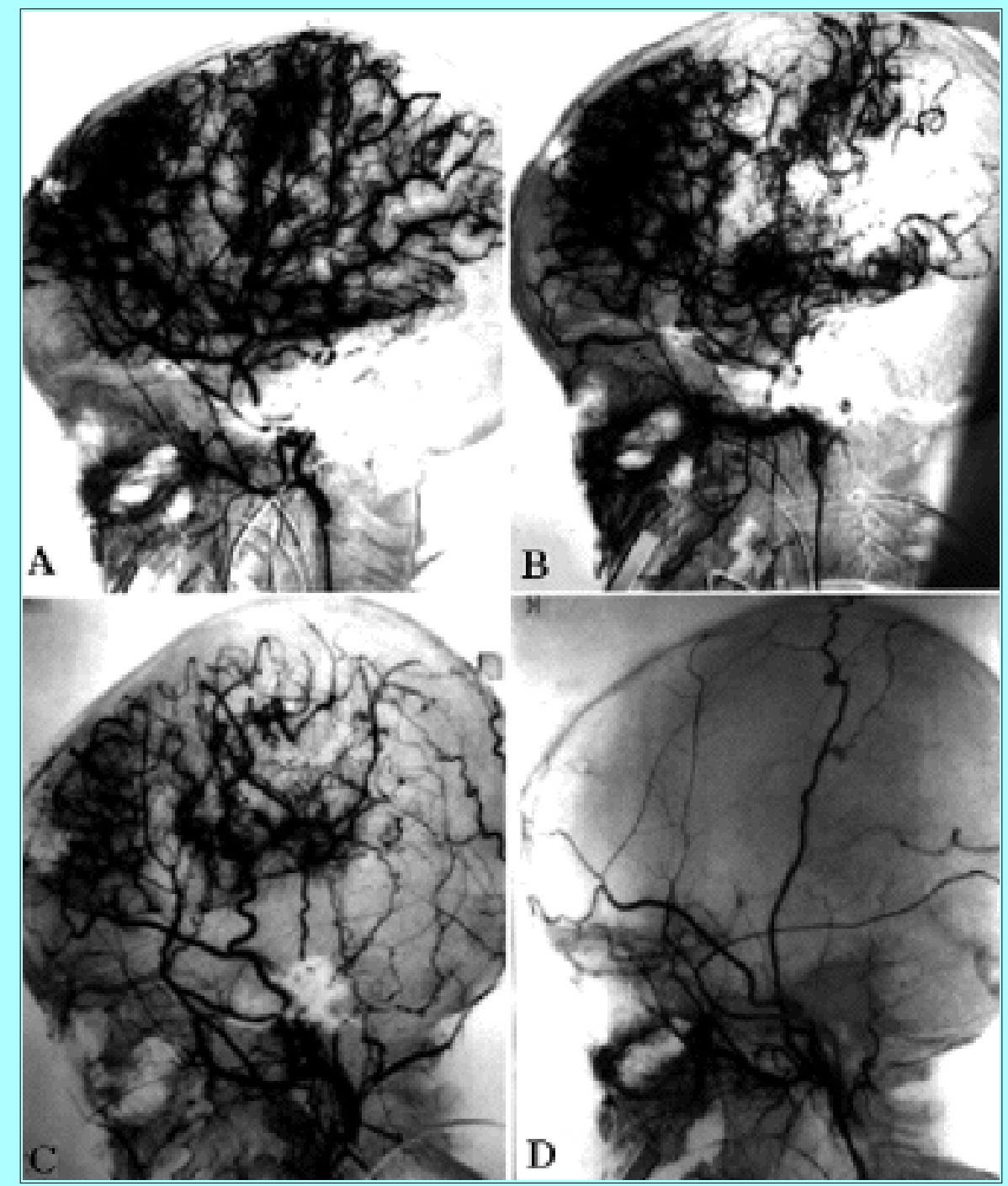

Fig. 3. Lateral-view angiograms showing of the extent of postoperative collateral formation. The angiograms demonstrate whole MCA (A), over two-thirds MCA (B), over one-third MCA (C), and no MCA distribution. In A, B, and C, collateral vessel formations were observed in both the MCA and ACA distributions. Only the extent of the MCA distribution was evaluated. Angiograms used. [With permission from Suzuki O., et al., (26)]

\section{Surgical Techniques}

In the original EDAS-alone procedure (Fig. 1 left), we performed the surgery reported by Matsushima, and coworkers.[22,23] In the STA-MCA anastomosis using EMS (Fig. 1 right), after STA-MCA anastomosis, the exposed cortex was covered by the temporal muscle as described by Karasawa, et 
al.[4,6] In the MCI procedure, we used three different indirect bypass techniques with two craniotomies on one side, while performing EDAS and EMS in the temporoparietal region and EMAS in the frontal region, as already reported (Fig. 2).[15,19] The posterior branch of the STA and the temporal muscle were utilized for the EDAS and the EMS, whereas the anterior branch of the STA and the frontal muscle with the periosteum were used for the EMAS.

\section{RESULTS}

Collateral formation occurred in over two-thirds of the MCA distribution in eight of 18 hemispheres (44\%), 18 of 35 hemispheres (52\%), and 14 of 19 hemispheres (74\%) treated by EDAS alone, MCI procedure, and direct anastomosis, respectively (Table 2). After undergoing the MCI procedure and direct anastomosis, collateral formation of the whole MCA distribution was observed in seven of 35 (20\%) and in four of 19 hemispheres (21\%), respectively. Furthermore, the MCI procedure resulted in well-formed collaterals in the ACA distribution in 33 of 35 hemispheres (94\%). On the other hand, the EDAS alone showed no collateral formation in $17 \%$ and collaterals in the whole MCA in $0 \%$ of treated hemispheres. Direct anastomosis produced postoperative collateral formation in all cases; and one or two of the frontal EMAS, as well as the temporoparietal EDAS and EMS in the MCI procedure produced good collateral formation. Direct anastomosis was thus found to be definitely superior to the two indirect procedures in demonstrating collateral formation. The MCI procedure showed a more extensive collateral formation than the EDAS alone, because it formed collaterals in the ACA distribution in addition to the MCA distribution.

\begin{tabular}{|c|c|c|c|}
\hline \multirow[b]{3}{*}{$\begin{array}{l}\text { Collat eal Formation } \\
\text { in the MCA }\end{array}$} & \multicolumn{3}{|c|}{$\begin{array}{l}\text { TABLE } 2 \\
\text { F THE COLLATERAL FORMATION IN THE MCA } \\
\text { HE THREE DIFFERENT PROCEDURES }\end{array}$} \\
\hline & \multicolumn{3}{|c|}{ No. of Hemisp heres Treated (\%) } \\
\hline & $\begin{array}{l}\text { EDAS } \\
\text { Alone }\end{array}$ & $\begin{array}{l}\text { Multiple } \\
\text { Combined }\end{array}$ & Dir ect \\
\hline $\begin{array}{l}\text { whole } \\
\text { ower two-thirds } \\
\text { ower one-thirds } \\
\text { none } \\
\text { totel }\end{array}$ & $\begin{array}{c}0(0) \\
8(44) \\
7(39) \\
3(17) \\
18(100)\end{array}$ & $\begin{array}{c}7(20) \\
11(32) \\
12(34) \\
5(14) \\
35(100)\end{array}$ & $\begin{array}{r}4(21) \\
10(53) \\
5(26) \\
0(0) \\
19(100)\end{array}$ \\
\hline
\end{tabular}

Clinical symptoms improved in all patients after undergoing the direct procedure and resolved completely in $74 \%$ (Table 3). Symptoms resolved completely in $63 \%$ and $56 \%$ after the MCI procedure and EDAS alone, respectively. Symptoms remained unchanged in 5\% of patients 1 year after undergoing EDAS alone and in $9 \% 1$ year after undergoing the MCI procedure. 


\begin{tabular}{|c|c|c|c|}
\hline \multicolumn{4}{|c|}{$\begin{array}{c}\text { TABLE } 3 \\
\text { COMPARISON OF IMPROVED CL NICAL STATUS AMONG PATIENTS } \\
\text { UNDERGOING THE THREE DIFFERENT PROCEDURES }\end{array}$} \\
\hline \multirow[b]{2}{*}{ Symptorns } & \multicolumn{3}{|c|}{ No. of Hemisp heres Treated (\%) } \\
\hline & $\begin{array}{l}\text { EDAS } \\
\text { Alone }\end{array}$ & $\begin{array}{l}\text { Multiple } \\
\text { Combined }\end{array}$ & Dir ext \\
\hline $\begin{array}{l}\text { com pletely disappeared } \\
\text { decre ased } \\
\text { unchanged } \\
\text { total }\end{array}$ & $\begin{array}{l}10(56) \\
7(39) \\
1(5) \\
18(100)\end{array}$ & $\begin{array}{l}22(63) \\
10(28) \\
3(9) \\
35(100)\end{array}$ & $\begin{array}{c}14(74) \\
5(26) \\
0(0) \\
19(100)\end{array}$ \\
\hline
\end{tabular}

There was only one minor stroke (6\%) after EDAS alone was performed, two epidural hematomas (6\%) after the MCI procedure, and one major (5\%) and one minor stroke (5\%) after the direct anastomosis was performed (Table 4). In the two cases of epidural hematoma reoperation was required; however, all neurological deficits resolved within a few weeks of operation. In the patient who suffered the major stroke after undergoing the direct anastomosis, not only the anastomotic portion but also several other branches of the MCA were occluded several days postsurgery, and the patient experienced hemiparesis even after rehabilitation. None of the strokes seemed to be the result of anesthetic complication.

\begin{tabular}{|c|c|c|c|}
\hline $\begin{array}{l}\text { COMPARISON OF } \\
\text { AMONG PATIENTS UN }\end{array}$ & $\begin{array}{r}\text { TABL } \\
\text { CAL AND F } \\
\text { SOING THE }\end{array}$ & $\begin{array}{l}\text { PERATIVE C } \\
\text { E DIFFEREN }\end{array}$ & $\begin{array}{l}\text { CATONS } \\
\text { CEDURES }\end{array}$ \\
\hline & No. & mispheres & $(\%)$ \\
\hline Complication & $\begin{array}{l}\text { EDAS } \\
\text { Alone }\end{array}$ & $\begin{array}{l}\text { Multiple } \\
\text { Combined }\end{array}$ & Dir ext \\
\hline $\begin{array}{l}\text { minor stroke } \\
\text { major stroke } \\
\text { hematoma } \\
\text { conwulsion } \\
\text { bone flap infection } \\
\text { totil }\end{array}$ & $\begin{array}{c}1(6) \\
0(0) \\
0(0) \\
1(6) \\
1(6) \\
18(100)\end{array}$ & $\begin{array}{c}0(0) \\
0(0) \\
2(6) \\
0(0) \\
1(3) \\
35(100)\end{array}$ & $\begin{array}{c}1(5) \\
1(5) \\
0(0) \\
0(0) \\
0(0) \\
19(100)\end{array}$ \\
\hline
\end{tabular}

\section{DISCUSSION}

Various bypass procedures have been performed in the treatment of moyamoya disease.[2-5,7,8,15,16,19,21,22,27,29] Direct anastomosis procedures such as STA-MCA bypass form collateral vessels with a high reliability, but these procedures are often technically difficult to perform. $[4,5,18,24]$ Small pediatric patients often do not have a large enough recipient cerebral artery to allow for anastomosis. The cerebral arteries in these patients are usually fragile. On the other hand, although indirect nonanastomosis procedures are safe and easy, they do have several drawbacks.[12-14,16,18,20,24] They do not always form sufficient collateral vessels , $[13,14]$ and even if vessels do form postoperatively, the cerebral circulation and metabolism tend only to improve near the operative field.[1,10,14] In such cases reoperation may be required.[12,20,24,30] We therefore gradually changed from performing a single-procedure indirect bypass to a multiple-procedure indirect bypass and from a single operative field to multiple fields that extend into the ACA and/or posterior cerebral artery (PCA) distribution areas. Consequently the frontotemporoparietal combined indirect bypass procedure has become our MCI procedure of choice.[11,15,16-19] 
In this study we compared the postoperative collateral formation, clinical improvement, and complications of these three different procedures. A positron emission tomography study, which can demonstrate postoperative decreases in the perfused area, seems to be the best measure to estimate postoperative improvement in the cerebral circulation.[1,14] However, it is difficult to perform in small children. Because postoperative collateral vessel formation is relatively related to the improvement in cerebral circulation, we examined the collateral formation instead of performing positron emission tomography studies.

Our results showed that, of the three procedures, direct anastomosis provided the best postoperative collateral formation and clinical improvement. However, surgical procedures should be evaluated not only for collateral formation but also for technical difficulties and postoperative complications. Of 16 patients in whom 19 hemispheres were treated, one patient suffered a major stroke after undergoing direct anastomoses. When complications occur in direct anastomosis procedures, they tend to be severe. In patients with moyamoya disease a few branches of the MCA may be easily occluded as an extension of an occlusion of the stenotic anastomotic portion. In addition, performing direct anastomosis in pediatric patients with moyamoya disease is more difficult than in adult patients with arteriosclerotic occlusive disease. Therefore, direct anastomosis should only be performed by skilled neurosurgeons who are familiar with the procedure.

In the frontotemporoparietal combined indirect bypass procedure, one or two of the three techniques formed sufficient collateral vessels with relatively high reliability not only in the MCA but also in the ACA regions. Furthermore, the procedure resulted in a better collateral formation than the original EDAS alone. The MCI procedure seems to be superior to any single procedure. In some cases in which a single indirect procedure is performed, an additional operation or a reoperation has been required.[12,20,24,29] Matsushima, et al.,[21] who developed the original EDAS by using the posterior branch of the STA, later reported a dual EDAS using both posterior and anterior branches of the STA to create a more extensive indirect anastomosis procedure, and Tenjin, et al.,[29] reported performing multiple EDAS procedures. On the other hand, we must remember that complications such as hematoma can still occur even after indirect procedures.[25] In our experience, the MCI procedure seems to cause hematoma more frequently than the original EDAS- alone procedure.

Both direct anastomosis and the MCI procedure are performed to correct ischemia in the anterior circulation; however, the effects of ischemia still remains in the PCA distribution and may even lead to visual disturbance.[6,28] Consequently, in both procedures, the temporoparietal craniotomy should be opened slightly wider posteriorly so that the EMS can reach the cortex of the PCA territory. Several authors have reported performing additional procedures for the treatment of posterior circulation ischemia.[27,29]

At present, we believe that the proper selection of the procedure depends on the patient's age and that a combination of indirect bypass procedures, such as ours, is therefore the first choice in treating pediatric patients younger than 5 years of age. This procedure is safe, easy, and also covers a wide area of the ischemic brain while demonstrating a high reliability. However, the extent of collateral formation after the same bypass procedure varies considerably from patient to patient and from area to area. It seems wiser, therefore, to design surgical fields after mapping the misery perfusion area when possible.[1,10,14]

\section{Acknowledgments}


We express our gratitude to Professor Emeritus Katsutoshi Kitamura, Neurological Institute, Faculty of Medicine, Kyushu University, for his continuing valuable suggestions. We are also grateful to Drs. Yasuo Kuwabara, Kanehiro Hasuo and Futoshi Mihara, Department of Radiology, for their valuable assistance in the examinations. This work was supported in part by research grants from the Ministry of Health and Welfare in Japan.

\section{References}

1. Ikezaki K, Matsushima T, Kuwabara Y, et al: Cerebral circulation and oxygen metabolism in childhood moyamoya disease: a perioperative positron emission tomography study. J Neurosurg 81:843-850, 1994

2. Inoue T, Matsushima T, Nagata S, et al: [Frontal encephalo-myo-arterio-synangiosis (EMAS).] Surg Cereb Stroke 20:297-300, 1992 (Jpn)

3. Karasawa J, Kikuchi H, Furuse S, et al: A surgical treatment of "moyamoya" disease--"encephalo-myo-synangiosis." Neurol Med Chir 17:29-37, 1977

4. Karasawa J, Kikuchi H, Furuse S, et al: Treatment of moyamoya disease with STA-MCA anastomosis. J Neurosurg 49:679-688, 1978

5. Karasawa J, Touho H, Ohnishi H, et al: Cerebral revascularization using omental transplantation for childhood moyamoya disease. J Neurosurg 79:192-196, 1993

6. Karasawa J, Touho H, Ohnishi H, et al: Long-term follow-up study after extracranial-intracranial bypass surgery for anterior circulation ischemia in childhood moyamoya disease. J Neurosurg 77:84-89, 1992

7. Kashiwagi S, Kato S, Yasuhara S, et al: Use of a split dura for revascularization of ischemic hemispheres in moyamoya disease. J Neurosurg 85:380-383, 1996

8. Kinugasa K, Mandai S, Kamata I, et al: Surgical treatment of moyamoya disease: operative technique for encephalo-duro-arterio-myo-synangiosis, its follow-up, clinical results, and angiograms.

Neurosurgery 32:527-531, 1993

9. Kurokawa T, Tomita S, Ueda K, et al: Prognosis of occlusive disease of the circle of Willis (Moyamoya disease) in children. Pediatr Neurol 1:274-277,1985

10. Kuwabara Y: [Evaluation of $\mathrm{CBF}, \mathrm{OEF}, \mathrm{CMRO} 2$ and mean transit time in moyamoya disease using positron emission computed tomography.] Jpn J Nucl Med 23:1381-1402,1986 (Jpn)

11. Matsushima T: [Moya-moya disease: its physiopathology and surgical treatment.] Fukuoka Acta Med 85:277-281,1994 (Jpn)

12. Matsushima T, Fujiwara S, Nagata S, et al: Reoperation for moyamoya disease refractory to encephalo-duro-arterio-synangiosis. Acta Neurochir 107:129-132, 1990

13. Matsushima T, Fujiwara S, Nagata S, et al: Surgical treatment for paediatric patients with Moyamoya disease by indirect revascularization procedures (EDAS, EMS, EMAS). Acta Neurochir 98:135-140, 1989 
14. Matsushima T, Fukui M, Kitamura K, et al: Encephalo-duro-arterio-synangiosis in children with moyamoya disease. Acta Neurochir 104:96-102, 1990

15. Matsushima T, Inoue T, Ikezaki K, et al: [Fronto-temporo-parietal combined indirect bypass for children with moyamoya disease. Part 1: Surgical procedure and techniques.] Nerv Syst Child 20:317-321, 1995 (Jpn)

16. Matsushima T, Inoue T, Katsuta T, et al: An indirect revascularization method in the surgical treatment of Moyamoya disease--various kinds of indirect procedures and a multiple combined indirect procedure. Neurol Med Chir Suppl 38:297-302,1998

17. Matsushima T, Inoue T, Suzuki SO, et al: [Combined indirect double bypass procedure for children with Moyamoya disease--Kyushu University method.] Video J 2:4, 1994 (Jpn)

18. Matsushima T, Inoue T, Suzuki SO, et al: Surgical treatment of moyamoya disease in pediatric patients--comparison between the results of indirect and direct revascularization procedures.

Neurosurgery 31:401-405, 1992

19. Matsushima T, Inoue TK, Suzuki SO, et al: Surgical techniques and the results of a frontotemporoparietal combined indirect bypass procedure for children with moyamoya disease: a comparison with the results of encephalo-duro-arterio-synangiosis alone. Clin Neurol Neurosurg 99 (Suppl 2):S123-S127, 1997

20. Matsushima Y, Aoyagi M, Nagaoka T, et al: [Children with moyamoya disease who needed additional indirect operations after encephalo-duro-arterio-synangiosis (EDAS).] Nerv Syst Child 19:403-410, 1994 (Jpn)

21. Matsushima Y, Aoyagi M, Suzuki R, et al: [Dual anastomosis for pediatric moyamoya patients using the anterior and the posterior branches of the superficial temporal artery.] Nerv Syst Child 18:27-32, 1993 (Jpn)

22. Matsushima Y, Fukai N, Tanaka K, et al: A new surgical treatment of moyamoya disease in children: a preliminary report. Surg Neurol 15:313-320, 1981

23. Matsushima Y, Inaba Y: Moyamoya disease in children and its surgical treatment. Introduction of a new surgical procedure and its follow-up angiograms. Childs Brain 11:155-170, 1984

24. Miyamoto S, Kikuchi H, Karasawa J, et al: Pitfalls in the surgical treatment of moyamoya disease. Operative techniques for refractory cases. J Neurosurg 68:537-543, 1988

25. Sonobe M, Takahashi S, Kubota Y, et al: [Chronic subdural hematoma developing after EMS for moyamoya disease.] Neurol Surg 10:857-859,1982 (Jpn)

26. Suzuki SO, Matsushima T, Inoue T, et al: [Fronto-temporo-parietal combined indirect bypass procedures for children with moyamoya disease. Part 2: Surgical results.] Nerv Syst Child 20:322-326, 1995 (Jpn)

27. Takahashi A, Kamiyama H, Houkin K, et al: Surgical treatment of childhood moyamoya disease--comparison of reconstructive surgery centered on the frontal region and the parietal region.

Neurol Med Chir 35:231-237, 1995 
28. Tashima-Kurita S, Matsushima T, Kato M, et al: Moyamoya disease. Posterior cerebral artery occlusion and pattern-reversal visual-evoked potential. Arch Neurol 46:550-553,1989

29. Tenjin H, Ueda S: Multiple EDAS (encephalo-duro-arterio-synangiosis). Additional EDAS using the frontal branch of the superficial temporal artery (STA) and the occipital artery for pediatric moyamoya patients in whom EDAS using the parietal branch of STA was insufficient. Childs Nerv Syst 13:220-224, 1997

30. Touho H, Karasawa J, Ohnishi H, et al: Surgical reconstruction of failed indirect anastomosis in childhood Moyamoya disease. Neurosurgery 32:935-940,1993

Manuscript received September 22, 1998.

Accepted in final form October 12, 1998.

Reprint requests: Toshio Matsushima, M.D., Department of Neurosurgery, Neurological Institute, Faculty of Medicine, Kyushu University 60, 3-1-1 Maidashi, Higashi-ku, Fukuoka 812, Japan. 